\title{
FINITELY ADDITIVE SUPERMARTINGALES ARE DIFFERENCES OF MARTINGALES AND ADAPTED INCREASING PROCESSES
}

\author{
THOMAS E. ARMSTRONG
}

\begin{abstract}
It is shown that any nonnegative bounded supermartingale admits a Doob-Meyer decomposition as a difference of a martingale and an adapted increasing process upon appropriate choice of a reference probability measure which may be only finitely additive.
\end{abstract}

Introduction. In [Armstrong, 1983] it is shown that every bounded finitely additive supermartingale is a decreasing process with respect to some reference probability measure $P$. This concept of a decreasing (or increasing) process is weaker than that corresponding to decreasing (increasing) processes of random variables adapted to a filtration. The corresponding class of finitely additive processes are called adapted decreasing (increasing) processes with respect to $P$. Theorem $B$ asserts that for every bounded nonnegative finitely additive supermartingale $Y$ there is a probability $P$ so that $Y=M-A$ where $M$ is a martingale and $A$ is an adapted increasing process with respect to $P$. In order to establish this it is necessary in Proposition A to show that for $g=\left\{g_{t}: t \in T\right\}$ an ordinary $L^{1}$-bounded nonnegative supermartingale adapted to the linearly ordered filtration $\left\{\mathscr{F}_{t}: t \in T\right\}$ on the probability measure space $(X, \mathscr{F}, P)$ to be expressed as the difference $m-a$ where $m$ is a martingale and $a$ is an increasing process with $0=\inf _{t} a_{t}$ it is necessary and sufficient that $\left\{g_{\tau}\right.$ : $\tau$ simple $T$-valued stopping time $\leqslant t\}$ be uniformly integrable for all $t \in T$. This extends the usual Doob-Meyer Decomposition Theorem in allowing arbitrary linearly ordered $T$.

Finitely additive supermartingales are differences of martingales and increasing adapted sequences. The Doob-Meyer Decomposition Theorem asserts that a nonnegative $L^{1}$-bounded supermartingale $f=\left\{f_{t}: 0 \leqslant t<\infty\right\}$ adapted to a filtration $\left\{\mathscr{F}_{i}: 0 \leqslant t<\infty\right\}$ of sub- $\sigma$-algebras in a probability space $(X, \mathscr{F}, P)$ admits a decomposition $f=m-a$ where $m$ is a martingale and $a$ is an increasing process with $a_{0}=0$ iff $f$ is of class DL. We recall that $f$ is of class DL iff $\left\{f_{\tau}: \tau\right.$ stopping time $\leqslant t\}$ is uniformly integrable (i.e., $\sigma\left(L^{1}, L^{\infty}\right)$-relatively compact) for each $t \in$ $[0, \infty)$. Attention may be confined to stopping times $\tau$ with only finitely many

Received by the editors May 23, 1983 and, in revised form, April 16, 1984 and February 12, 1985.

1980 Mathematics Subject Classification. Primary 60G48, 28A60.

Key words and phrases. Martingale, supermartingale, increasing process, finitely additive measure, set function process. 
values (i.e., simple stopping times) in the definition of class DL. Usually the Doob-Meyer Decomposition Theorem is stated for right-continuous $f$ but holds for arbitrary $f$ as is shown in Theorem 20 of Appendix I of Dellacherie and Meyer [1982]. See Mertens [1971] for one of the first results in this vein. Imposing predictability guarantees uniqueness on the increasing process but this will not be of concern to us here.

In Armstrong [1983] finitely additive supermartingales were introduced. See Gut and Schmidt [1983] for Schmidt's work on finitely additive supermartingales on the integers. The topic of concern here is the Doob-Meyer Decomposition Theorem in this context. We recall that in Armstrong [1983] filtrations of $\sigma$-algebras are replaced by linearly ordered families of subalgebras of a Boolean algebra $\mathscr{B}$. If $\Gamma$ is a chain of subalgebras of $\mathscr{B}$ then a process $Y$ on $\Gamma$ is a family $\left\{Y_{\mathscr{A}}: \mathscr{A} \in \Gamma\right\}$ where each $Y_{\mathscr{A}}$ is a finitely additive measure of bounded variation on the subalgebra $\mathscr{A}$ of $\mathscr{B}$. Suppose that $\mathscr{B}$ is the $\sigma$-algebra $\mathscr{F}$ of a probability space $(X, \mathscr{F}, P), \Gamma$ is a filtration $\left\{\mathscr{F}_{t}: 0 \leqslant t<\infty\right\}$, and that $f=\left\{f_{t}: 0 \leqslant t<\infty\right\}$ is an $L^{1}$-bounded stochastic process adapted to $\Gamma$. In this case one obtains a process $Y$ on $\Gamma$ by setting $Y_{t}$ equal to that measure on $\mathscr{F}_{t}$ with $P$-density $f_{t}$. Note that $E\left(f_{s} \mid \mathscr{F}_{t}\right)$ corresponds to restricting $Y_{s}$ to $\mathscr{F}_{t}$. As a result we adopt the conditional expectation notation $E(\mu \mid \mathscr{A})$ for the restriction to $\mathscr{A}$ of a finitely additive $\mu$ on a superalgebra of $\mathscr{A}$. One important distinction between processes of finitely additive measures and stochastic processes of random variables is the absence of a reference probability measure. This makes quite a bit of difference in what follows.

A process $Y$ on a chain $\Gamma$ of subalgebras of $\mathscr{B}$ is said to be a supermartingale provided that when $\mathscr{A}_{1} \subset \mathscr{A}_{2}$ are in $\Gamma$ then $Y_{\mathscr{A}_{1}} \geqslant E\left(Y_{\mathscr{A}_{2}} \mid \mathscr{A}_{1}\right) . Y$ is said to be a martingale if $Y_{\mathscr{A}_{1}}=E\left(Y_{\mathscr{A}_{2}} \mid \mathscr{A}_{1}\right)$ in the same circumstance. Given a supermartingale $Y$ one wishes to find a decomposition $Y=M-A$ where $M$ is a martingale and $A$ is an "increasing process" with $\inf \left\{\left\|A_{\mathscr{A}}\right\|: \mathscr{A} \in \Gamma\right\}=0$. One definition of increasing process is given in Armstrong [1983]. If $\left\{\mu_{\mathscr{A}}: \mathscr{A} \in \Gamma\right\}$ is a family in $\mathrm{BA}(\mathscr{B})$ so that $\mu_{\mathscr{A}_{1}} \leqslant \mu_{\mathscr{A}_{2}}$ if $\mathscr{A}_{1} \subset \mathscr{A}_{2}$ then this family is said to be increasing and $Y=\left\{E\left(\mu_{\mathscr{A}} \mid \mathscr{A}\right)\right.$ : $\mathscr{A} \in \Gamma\}$ is called an increasing process on $\Gamma$. Decreasing processes are analogously defined. It is shown in $\S 6$ of Armstrong [1983] that every supermartingale $Y$ does admit a decomposition $Y=M-A$ where $M$ is a martingale and $A$ is increasing with $\inf \left\{\left\|A_{\mathscr{A}}\right\|: \mathscr{A} \in \Gamma\right\}=0$. In fact, $Y$ is a decreasing process. This is used to show that the class of differences of nonnegative finitely additive supermartingales (i.e., $F$-processes) is the class of differences of nonnegative submartingales. One consequence is the existence of at least one $P$ in $\mathrm{BA}_{1}^{+}(\mathscr{B})$ (the finitely additive probability measures on $\mathscr{B})$ so that $Y_{\mathscr{A}} \ll E(P \mid \mathscr{A})$ for all $\mathscr{A} \in \Gamma$. Here $\ll$ denotes the $\varepsilon-\delta$ notion of absolute continuity standard for finitely additive measures. Unfortunately, the increasing processes are not the precise analogue of increasing stochastic processes adapted to a filtration but are more general as well be seen.

One may convert finitely additive processes on a chain $\Gamma$ of subalgebras of $\mathscr{B}$ into countably additive processes on a filtration of $\sigma$-algebras. This may be done by passage to the totally disconnected compact Hausdorff Stone space $X_{\mathscr{B}}$. The clopen algebra of $X_{\mathscr{B}}$ is isomorphic to $\mathscr{B}$ under a map $A \rightarrow[A]$ from $\mathscr{B}$ to the clopen 
algebra $[\mathscr{B}]$. If $\mathscr{A}$ is a subalgebra of $\mathscr{B}$ then $\mathscr{A}$ is isomorphic to $[\mathscr{A}]=\{[A]$ : $A \in \mathscr{A}\} \subset \mathscr{B}$. Denote by $\mathscr{F}_{\mathscr{B}}$ the Baire algebra $\sigma([\mathscr{B}])$ of $X_{\mathscr{B}}$. For $\mathscr{A}$ a subalgebra of $\mathscr{B}$ denote by $\mathscr{F}_{\mathscr{A}}$ the $\sigma$-algebra $\sigma([\mathscr{A}])$. It may be shown that $\mathscr{F}_{\mathscr{A}}$ is isomorphic to the Baire algebra of the Stone space $X_{\mathscr{A}}$ of $\mathscr{A}$ upon identifying [ $A$ ] or $A$ with the corresponding clopen set in $X_{\mathscr{A}}$. The Stone correspondence identifies a $\mu \in \operatorname{BA}(\mathscr{B})$ with a unique $\tilde{\mu} \in \mathscr{M}\left(X_{\mathscr{B}}\right)$ (the Radon measures on $X_{\mathscr{B}}$ ) via the formula $\mu(A)=$ $\tilde{\mu}([A])$ for all $\mathscr{A} \in \mathscr{B}$. The map $\mu \rightarrow \tilde{\mu}$ is a Banach lattice isomorphism from $\operatorname{BA}(\mathscr{B})$ onto $\mathscr{M}\left(X_{\mathscr{B}}\right) . \mathscr{M}\left(X_{B}\right)$ is considered to be $\mathrm{CA}\left(\mathscr{F}_{\mathscr{B}}\right)$, the countably additive Baire measures on $X_{\mathscr{B}}$, in the usual fashion. If $\mu$ is in $\operatorname{BA}(\mathscr{A})$ for some subalgebra $\mathscr{A}$ of $\mathscr{B}$ then $\tilde{\mu}$ is defined in the same manner as above as an element of $\mathrm{CA}\left(\mathscr{F}_{\mathscr{A}}\right)$. It is easily seen that if $Y=\left\{Y_{\mathscr{A}}: \mathscr{A} \in \Gamma\right\}$ is a process on a chain $\Gamma$ of subalgebras of $\mathscr{B}$ then $\tilde{Y}=\left\{\tilde{Y}_{\mathscr{A}}: \mathscr{A} \in \Gamma\right\}$ is a countably additive process on $\left\{\mathscr{F}_{\mathscr{A}}: \mathscr{A} \in \Gamma\right\}$ and that all countably additive processes on $\left\{\mathscr{F}_{\mathscr{A}}: \mathscr{A} \in \Gamma\right\}$ arise in this fashion. A process $Y$ is a martingale, supermartingale or increasing process iff $\tilde{Y}$ is. $Y$ is such that $Y_{\mathscr{A}} \ll$ $E(P \mid \mathscr{A})$ for all $\mathscr{A}$ for some $P \in \mathrm{BA}_{1}^{+}(\mathscr{B})$ iff $\tilde{Y}_{\mathscr{A}} \ll E\left(\tilde{P} \mid \mathscr{F}_{\mathscr{A}}\right)$ for all $\mathscr{A}$. In this case, let $f_{\mathscr{A}}^{P}$ be the density of $\tilde{Y}_{\mathscr{A}}$ with respect to $E\left(\tilde{P} \mid \mathscr{F}_{\mathscr{A}}\right)$ for all $\mathscr{A}$. The family $f^{P}=\left\{f_{\mathscr{A}}^{P}: \mathscr{A} \in \Gamma\right\}$ is a stochastic process adapted to the filtration $\left\{\mathscr{F}_{\mathscr{A}}: \mathscr{A} \in \Gamma\right\}$ which is a supermartingale, martingale, increasing process iff $Y$ is.

An increasing process $A=\left\{A_{\mathscr{A}}: \mathscr{A} \in \Gamma\right\}$ is said to be increasing with respect to $P \in \mathrm{BA}_{1}^{+}(\mathscr{B})$ iff it is of the form $\left\{E\left(\mu_{\mathscr{A}} \mid \mathscr{A}\right): \mathscr{A} \in \Gamma\right\}$ with $\left\{\mu_{\mathscr{A}}: \mathscr{A} \in \Gamma\right\}$ an increasing family of measures absolutely continuous to $P$. If $g_{\mathscr{A}}^{P}$ is the $\tilde{P}$-density of $\tilde{\mu}_{\mathscr{A}}$ we have $g^{P}=\left\{g_{\mathscr{A}}^{P}: \mathscr{A} \in \Gamma\right\}$ increasing in $L^{1}\left(X_{\mathscr{B}}, \mathscr{F}_{\mathscr{B}}, \tilde{P}\right)$. It need not be the case that $g^{P}$ is adapted to $\left\{\mathscr{F}_{\mathscr{A}}: \mathscr{A} \in \Gamma\right\}$. If this is the case we say that $A$ is an adapted increasing process with respect to $P$. An equivalent requirement is that $h^{P}=\left\{E\left(g_{\mathscr{A}}^{P} \mid \mathscr{F}_{\mathscr{A}}\right): \mathscr{A} \in \Gamma\right\}$ be increasing in $L^{1}\left(X_{\mathscr{D}}, \mathscr{F}_{\mathscr{B}}, \tilde{P}\right)$, for $\left\{\mu_{\mathscr{A}}: \mathscr{A} \in \Gamma\right\}$ could be replaced by $\left\{\nu_{\mathscr{A}}: \mathscr{A} \in \Gamma\right\}$ where $\tilde{\nu}_{\mathscr{A}}=h_{\mathscr{A}}^{P} \tilde{P}$ for $\mathscr{A} \in \Gamma$. One may give a necessary and sufficient condition purely in terms of the finitely additive measures $\left\{\mu_{\mathscr{A}}: \mathscr{A} \in \Gamma\right\}$ and $P$ so that $A$ be adapted. This is that each $\mu_{\mathscr{A}}$ is the limit in variation norm of a sequence $\left\{\mu_{\mathscr{\infty}}^{n}: n \in N\right\}$ where each $\mu_{\mathscr{A}}^{n}$ is obtained from $P$ by choosing a finite partition $\left\{A_{n j}: j=1, \ldots, m\right\} \subset \mathscr{A}$ scalars $\left\{\lambda_{n j}^{\mathscr{A}}: j=1, \ldots, m\right\}$ and letting $\mu_{\mathscr{A}}^{n}(B)=\sum_{j=1}^{m} \lambda_{n j}^{\mathscr{A}} P\left(B \cap A_{n j}\right)$. This is an exercise in the Bochner finitely-additive Radon-Nikodym theorem (Bochner and Phillips [1941]). We are nearly in a position to show that every finitely additive supermartingale is the difference of a martingale and an adapted increasing process with respect to some $P \in \mathrm{BA}_{1}^{+}(\mathscr{B})$. The only obstacle is the extension of Doob-Meyer Decomposition Theorem from filtrations indexed by $[0, \infty)$ to filtrations indexed by arbitrary linearly ordered set. This is not a great obstacle, for a supermartingale has its variation concentrated on an order separable set and such sets are isomorphic to subsets of $[0, \infty)$.

Proposition A. Let $\left\{\mathscr{F}_{t}: t \in T\right\}$ be a linearly ordered filtration in a probability space $(X, \mathscr{F}, P)$. In order that an $L^{1}$-bounded supermartingale $\left\{f_{t}: t \in T\right\}$ adapted to $\left\{\mathscr{F}_{t}: t \in T\right\}$ admit a decomposition $f=m-a$ where $m$ is a martingale and $a$ is an increasing process with $\inf \left\{\left\|a_{t}\right\|_{1}: t \in T\right\}=0$ it is necessary and sufficient that $\left\{f_{\tau}: \tau\right.$ simple $T$-valued stopping time $\leqslant t\}$ be uniformly integrable for all $t \in T$. 
Proof. The case where $T$ is order separable is considered first. In this case $T$ is order isomorphic to a subset of $[0, \infty)$ with $0=\inf T$. Regard $T$ as actually being a subset of $[0, \infty)$. Extend the filtration from $T$ to $[0, \infty)$ as follows. If $t \in[0, \infty)$, set $\mathscr{F}_{t}=\bigcap\left\{\mathscr{F}_{s}: t \leqslant s, s \in T\right\}$. Extend $f$ from $T$ to $[0, \infty)$ by setting $f_{t}=$ $\sup \left\{E\left(f_{s} \mid \mathscr{F}_{t}\right): t \leqslant s \in T\right\}$. We have $\left\{f_{\tau}: \tau\right.$ simple stopping time $\left.\leqslant t\right\}$ uniformly integrable for $0 \leqslant t<\infty$ iff $\left\{f_{\tau}: \tau\right.$ simple $T$-valued stopping time $\left.\leqslant t\right\}$ is uniformly integrable for $t \in T$. The reason for this is that uniform integrability of a family is preserved by adjoining to it arbitrary conditional expectations on sub- $\sigma$-algebras or sequential almost sure limits. Thus, if $\left\{f_{\tau}: \tau\right.$ simple $T$-valued stopping time $\left.\leqslant t\right\}$ is uniformly integrable for $t \in T$ then $f_{t}=m_{t}-a_{t}$ for $0 \leqslant t \leqslant \infty$ with $m$ a martingale and $a$ an increasing process with $a_{0}=0$. Restriction of $f, m$, and $a$ to $T$ gives us the desired decomposition. Conversely, if $f_{t}=m_{t}-a_{t}$ for $t \in T$ with $m$ a martingale and $a$ an increasing process with $\inf _{T} a_{t}=0$ then $\left\{m_{\tau}: \tau\right.$ simple stopping time $\left.\leqslant t\right\}$ is uniformly integrable for $t \in T$ and, since $\left\{a_{\tau}: \tau\right.$ simple stopping time $\left.\leqslant t\right\}$ is dominated by $a_{t}$, it is uniformly integrable for $t \in T$. As a result $\left\{f_{\tau}=m_{\tau}-a_{\tau}: \tau\right.$ simple stopping time $\leqslant t\}$ is uniformly integrable for $t \in T$. This establishes the order separable case.

In order to establish the proposition for nonseparable $T$ it is only necessary to show that if $\left\{f_{\tau}: \tau\right.$ simple $T$-valued stopping time $\left.\leqslant t\right\}$ is uniformly integrable for all $t \in T$ then $f$ admits a Doob-Meyer decomposition. The converse is established as in the preceding paragraph. Let $R \subset[0, \infty)$ denote $\left\{\left\|f_{t}\right\|_{1}: t \in T\right\}$. If $r \in \bar{R} \backslash R$ and $r$ is a limit from above of $\left\{\left\|f_{t}\right\|_{1}:\left\|f_{t}\right\|_{1}>r\right\}$, set $\mathscr{F}_{r^{-}}$equal to $\sigma\left\{\mathscr{F}_{t}:\left\|f_{t}\right\|_{1}>r\right\}$. If $r \in \bar{R} \backslash R$ is a limit from below of $\left\{\left\|f_{t}\right\|:\left\|f_{t}\right\|<r\right\}$, set $\mathscr{F}_{r^{+}}$equal to $\bigcap\left\{\mathscr{F}_{t}:\left\|f_{t}\right\|<r\right\}$. If $r \in R$ let $L_{r}=\left\{t \in T:\left\|g_{t}\right\|=r\right\}$. In this case set $\mathscr{F}_{r^{+}}$equal to $\boldsymbol{\sigma}\left\{\mathscr{F}_{t}:\left\|g_{t}\right\|=r\right\}$ and $\mathscr{F}_{r^{-}}$equal to $\bigcap\left\{\mathscr{F}_{t}:\left\|g_{t}\right\|=r\right\}$. Adjoin $\left\{r^{-}\right.$and $/$or $\left.r^{+}: r \in \bar{R}\right\}$ to $T$ to obtain $T^{\#}$ where $s \leqslant r^{-} \leqslant r^{+} \leqslant t$ if $\left\|g_{s}\right\|>r>\left\|g_{t}\right\|$ and $r^{-}<t<r^{+}$if $\left\|g_{t}\right\|=r$. The filtration $\left\{\mathscr{F}_{t}: t \in T^{\#}\right\}$, as defined, extends $\left\{\mathscr{F}_{t}: t \in T\right\}$. Since $\left\{f_{s}\right.$ : $s \leqslant t\}$ is uniformly integrable for $t \in T$ we may extend $f$ to be a supermartingale adapted to the filtration $\left\{\mathscr{F}_{t}: t \in T^{\sharp}\right\}$ by setting $f_{r^{-}}=\lim \left\{f_{t}:\left\|f_{t}\right\|>r\right\}$ or

$$
f_{r^{+}}=\lim \left\{f_{t}:\left\|f_{t}\right\|<r\right\}
$$

if $r \in \bar{R} \backslash R$ and by setting $f_{r^{-}}=\lim \left\{f_{t}: t\right.$ decreasing in $\left.L_{r}\right\}$ and

$$
f_{r^{+}}=\lim \left\{f_{t}: t \text { increasing in } L_{r}\right\}
$$

if $r \in R$. It is easily verified that $\left\{f_{\tau}: \tau\right.$ simple $T^{\sharp}$-valued stopping time $\left.\leqslant t\right\}$ is uniformly integrable for each $t \in T^{\sharp}$. The ensemble $\tilde{T}$ of all $r^{-}$or $r^{+}$for $r \in \bar{R}$ is obtained by deletion of $L_{r}$ from $T^{\#}$ for all $r \in R$. One may verify that if $D$ is a countable dense set in $R$ then $\left\{r^{+}, r^{-}: r \in D\right\}$ is a countable order dense set in $\tilde{T}$. One has $\left\{f_{\tau}: \tau\right.$ simple $\tilde{T}$-valued stopping time $\left.\leqslant t\right\}$ uniformly integrable for all $t \in \tilde{T}$. As a result, it may be concluded that, for all $t \in \tilde{T}, f_{t}=m_{t}-a_{t}$ where $m$ is a martingale and $a$ is an increasing process with $0=\inf \left\{a_{t}: t \in \tilde{T}\right\}$. Notice that if $r \in R$ then $a_{r^{-}}=a_{r^{+}}$almost surely for $f_{r^{-}}=f_{r^{+}}$almost surely. As a result, $a$ may be extended to $T^{\sharp}$ from $\tilde{T}$ as an increasing process. If $t \in L_{r}$ then $a_{t}$ must be the function $a_{r}$. Extend $m$ as a martingale from $\tilde{T}$ to $T^{\sharp}$. We have $f=m-a$ on $T^{\#}$. Upon restriction from $T^{\sharp}$ to $T$ we obtain the desired Doob-Meyer decomposition of $f$. This completes the proof of the proposition. 
THEOREM B. Let $Y$ be a nonnegative bounded finitely additive supermartingale on a chain $\Gamma$ of subalgebras of a Boolean algebra $\mathscr{B}$. There is a $P \in \mathrm{BA}_{1}^{+}(\mathscr{B})$ and a $Q \in \mathrm{BA}^{+}(\mathscr{B})$ with $Q \ll P$ so that if $\mathscr{M} Q$ is the martingale $\{E(Q \mid \mathscr{A}): \mathscr{A} \in \Gamma\}$ then $A=\mathscr{M} Q-Y$ is an adapted increasing process with respect to $P$.

Proof. By our previous remarks we know that $Y=M-A$ where $A$ is an increasing process with $\inf \left\{\left\|A_{\mathscr{A}}\right\|: \mathscr{A} \in \Gamma\right\}=0$ and $M$ is a martingale. We may find $\nu \in \mathrm{BA}^{+}(\mathscr{B})$ so that $E(\nu \mid \mathscr{A})=M_{\mathscr{A}}$ for $\mathscr{A} \in \Gamma$. Since $E(\nu \mid \mathscr{A})=M_{\mathscr{A}}$ it follows that $Y_{\mathscr{A}} \leqslant E(\nu \mid \mathscr{A})$ for $\mathscr{A} \in \Gamma$. Let $P$ be $\lambda \nu$ where $\lambda=\|\nu\|^{-1}$. It follows that $Y_{\mathscr{A}} \leqslant \lambda E(P \mid \mathscr{A})$ for $\mathscr{A} \in \Gamma$. Let us pass to the Stone space $X_{\mathscr{B}}$ equipped with the filtration $\left\{\mathscr{F}_{\mathscr{A}}: \mathscr{A} \in \Gamma\right\}$ as in previous remarks. Let $\tilde{P} \in \mathscr{M}_{1}^{+}(\mathscr{B})$ correspond to $P$ under the Stone correspondence. Let $\tilde{Y}=\left\{\tilde{Y}_{\mathscr{A}}: \mathscr{A} \in \Gamma\right\}$ be the countably additive supermartingale on $\left\{\mathscr{F}_{\mathscr{A}}: \mathscr{A} \in \Gamma\right\}$ corresponding to $Y$ under the Stone correspondence. Let $\left\{f_{\mathscr{A}}: \mathscr{A} \in \Gamma\right\}=f$ be the supermartingale adapted to $\left\{\mathscr{F}_{\mathscr{A}}: \mathscr{A} \in \Gamma\right\}$ with $\tilde{Y}_{\mathscr{A}}=f_{\mathscr{A}} E\left(\tilde{P} \mid \mathscr{F}_{\mathscr{A}}\right)$ for $\mathscr{A} \in \Gamma$. The supermartingale $f$ is uniformly bounded in $L^{\infty}$-norm by $\lambda$ hence is uniformly integrable. Proposition A may be applied to $f$ to yield a decomposition $f=m-a$ where $m$ is a martingale and $a$ is an increasing process with $\inf \left\{\left\|a_{\mathscr{A}}\right\|_{1}: \mathscr{A} \in \Gamma\right\}=0$. Since $m$ is uniformly integrable it is of the form $\left\{E\left(h \mid \mathscr{F}_{\mathscr{A}}\right): \mathscr{A} \in \Gamma\right\}$ for some $h \in L^{1+}\left(X_{\mathscr{B}}, \mathscr{F}_{\mathscr{B}}, \tilde{P}\right)$. Let $\tilde{Q}=h \tilde{P}$ and let $Q \in \mathrm{BA}^{+}(\mathscr{B})$ correspond to $\tilde{Q}$ under the Stone correspondence. If $\mathscr{A} \in \Gamma$ then $A_{\mathscr{A}}=E(Q \mid \mathscr{A})-Y_{\mathscr{A}}$ corresponds to $a_{\mathscr{A}} \tilde{P}$ under the Stone correspondence. It is immediate that this $Q$ is the one which we were seeking.

We may obtain the following as a nearly immediate corollary relating to ordinary supermartingales adapted to a filtration:

Corollary C. Let $(X, \mathscr{F}, P)$ be a probability measure space, $\left\{\mathscr{F}_{t}: t \in T\right\}$ be a

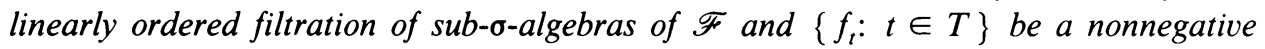
$L^{1}$-bounded supermartingale adapted to $\left\{\mathscr{F}_{t}: t \in T\right\}$. There is a $\Phi \in L_{1}^{\infty *+}(X, \mathscr{F}, P)$ and a $\Psi \in L^{\infty *+}(X, \mathscr{F}, P)$ with $\Psi \ll \Phi$ so that $A=\left\{A_{t}: \quad t \in T\right\} \subset$ $L^{\infty *+}(X, \mathscr{F}, P)$ defined by $A_{t}=E\left(\Psi \mid \mathscr{F}_{t}\right)-f_{t} P$ is an adapted increasing process with respect to $\Phi$.

Proof. $L^{\infty *}(X, \mathscr{F}, P)$ may be identified with $\operatorname{BA}\left(\mathscr{F}_{P}\right)$ where $\mathscr{F}_{P}$ is the measure algebra of $P$ obtained as the Boolean quotient of $\mathscr{F}$ by the ideal of $P$-negligible sets. The filtration $\left\{\mathscr{F}_{t}: t \in T\right\}$ induces, under the quotient map, a chain $\left\{\left(\mathscr{F}_{t}\right)_{P}: t \in T\right\}$ of subalgebras of $\mathscr{F}_{P}$. $\left\{f_{t} P: t \in T\right\}$ may be considered as a finitely additive supermartingale on this chain. Theorem B applies in this situation to establish the corollary.

ReMARKS. (a) Corollary C states that a Doob-Meyer decomposition exists for any supermartingale if we are allowed to change the reference probability measure from $P$ to an element $\Phi$ of $\mathrm{BA}_{1}^{+}\left(\mathscr{F}_{P}\right)$. Note that $\Phi$ is weakly absolutely continuous with respect to $P$ in annihilating all $P$-negligible sets. If $\Phi$ were to be countably additive it would be absolutely continuous with respect to $P$ hence of the form $h P$ for some $h \in L_{1}^{1+}(X, \mathscr{F}, P)$. In this case, $\Psi$ is of the form $g h P$ for some $g$ in $L^{1+}(X, \mathscr{F}, h P)$ and $A_{t}$ is of the form $a_{t} h P$ where $\left\{a_{t}: t \in T\right\}$ is an increasing process. In this case, 
it follows that $\left\{f_{\tau}: \tau\right.$-simple $T$-valued stopping time $\left.\leqslant t\right\}$ is uniformly integrable for $t \in T$. Thus, if $P=\Phi$ is an impossible choice for $\Phi$ then any suitable $\Phi$ must have a nontrivial purely finitely additive part. The work of Astbury [1981] is related to this.

(b) Another connection between finite additivity and the Doob-Meyer Decomposition Theorem is the characterization in Metivier and Pellaumail [1975] of those nonnegative supermartingales admitting such a decomposition as those whose Doleans-Föllmer measure on the ring of predictable sets is countably additive. Thus, those which do not admit such a decomposition have a nontrivial purely finitely additive part in their Doleans-Föllmer measure. The relationship between this observation and the remarks in (a) has yet to be made precise.

(c) It need not be the case that the Doob-Meyer decomposition for countably additive supermartingales leads one inevitably to finitely additive measures. Consider $\left\{X_{t}: t \in T\right\}$ a linearly ordered projective system of compact Hausdorff spaces with $P_{t, s}: X_{s} \rightarrow X_{t}$ the associated continuous surjection for $s \geqslant t$. Let $X_{\infty}$ be the projective limit with $P_{t}: X_{\infty} \rightarrow X_{t}$ the associated projection for $t \in T$. Let $\mathscr{F}$ be the Baire field of $X_{\infty}$ and for $t \in T$ let $\mathscr{B}_{t}$ be the Baire field of $X_{t}$. Let $\mathscr{F}_{t}=P_{t}^{-1}\left(\mathscr{B}_{t}\right) \subset \mathscr{F}$ for all $t \in T$. One may consider countably additive processes adapted to the filtration $\left\{\mathscr{F}_{t}: t \in T\right\}$. Equip $\mathrm{CA}^{+}\left(\mathscr{B}_{t}\right)$, hence $\mathrm{CA}^{+}\left(\mathscr{F}_{t}\right)$, with the vague topology so closed norm bounded sets are compact. The canonical surjection $\mathscr{A}_{t, s}: \mathrm{CA}^{+}\left(\mathscr{F}_{s}\right)$ $\rightarrow \mathrm{CA}^{+}\left(\mathscr{F}_{t}\right)$ associated with $P_{t, s}$ is vaguely continuous. The proof of Theorem 6-1 of Armstrong [1983] is valid in this setting as is that of Theorem B. As a result, if $Y$ is a countably additive norm bounded nonnegative supermartingale adapted to $\left\{\mathscr{F}_{t}\right.$ : $t \in T\}$ there is a $P \in \mathrm{CA}_{1}^{+}\left(\mathscr{F}_{\infty}\right)=\mathscr{M}_{1}^{+}\left(X_{\infty}\right)$, and a $Q \in \mathrm{CA}^{+}\left(\mathscr{F}_{\infty}\right)$ with $Q \ll P$ so that, if $M_{t} \in \mathrm{CA}^{+}\left(\mathscr{F}_{t}\right)$ is the restriction of $Q$ to $\mathscr{F}_{t}$ for all $t$, then $A_{t}=M_{t}-A_{t}$ for $t \in T$ yields an adapted increasing process with respect to $P$ with

$$
\inf \left\{\left\|A_{t}\right\|: t \in T\right\}=0 .
$$

(d) As we have remarked previously we have not considered uniqueness in the Doob-Meyer Decomposition Theorem. This is of considerable importance for the purpose of stochastic integration. If one considers supermartingales adapted to a linearly ordered filtration of sub- $\sigma$-algebras of a probability measure space it appears clear that the proof of Proposition A yields a predictable increasing process in the Doob-Meyer decomposition from a predictable increasing process on the order separable $\tilde{T}$ and conversely. Uniqueness of the predictable process on $T$ appears to follow from uniqueness on $\tilde{T}$. As a result it appears that, with the appropriate definition of predictability of finitely additive processes, there is a unique predictable adapted increasing process with respect to $P$ which occurs in a Doob-Meyer decomposition of a finitely additive supermartingale in Theorem B. Of course, different $P$ in Theorem A give rise to different predictable adapted increasing processes.

(e) The effects of change in the reference probability measures on semimartingales have been considered in several places. Memin [1980] is a notable example. Usually the new probability is one mutually absolutely continuous with respect to the original and in these cases always countably additive. 


\section{REFERENCES}

T. E. Armstrong [1983], Finitely additive F-processes, Trans. Amer. Math. Soc. 279, 271-295.

K. A. Astbury [1981], Order convergence of martingales in terms of countably additive and purely finitely additive martingales, Ann. Probab. 9, 226-275.

S. Bochner and R. S. Phillips [1941], Additive set functions and vector lattices, Ann. of Math. 42, 316-324.

C. Dellacheire and P. Meyer [1982], Probabilities and potential B. Theory of Martingales, NorthHolland, Amsterdam.

A. Gut and K. D. Schmidt [1983], Amarts and set function processes, Springer-Verlag, Berlin.

J. Memin [1980], Espaces de semi-martingales et changement de probabilité, Z. Wahrsch. Verw. Gebiete 52, 9-39.

J.-F. Mertens [1972], Théorie des processus stochastiques generaux applications aux supermartingales, Z. Warsch. Verw. Gebiete 22, 45-68.

M. Metivier and J. Pellaumail [1975], On Doleans-Föller measure for quasi-martingales, Illinois J. Math. 19, 491-504.

P. A. Meyer [1966], Probability and potential, Blaisdell, Waltham, Mass.

K. M. Rao [1969a], On decomposition theorems of Meyer, Math. Scand. 24, 66-78. [1969b], Quasi-martingales, Math. Scand. 24, 79-92.

Department of Theoretical Statistics, University of Minnesota, Minneapolis, Minnesota 55455

Current address: Department of Mathematics and Computer Science, University of Maryland-Balitmore County, Catonsville, Maryland 21228 